\title{
Heart rate variability in overactive bladder experimental model
}

\author{
Łukasz Dobrek, Piotr Thor
}

Pathophysiology Department, Jagiellonian University, Medical College, Cracow, Poland

Submitted: 25 July 2011

Accepted: 2 February 2012

Arch Med Sci 2013; 9, 5: 930-935

DOI: 10.5114/aoms.2012.30946

Copyright ๑ 2013 Termedia \& Banach
Corresponding author: Dr Łukasz Dobrek Department of Pathophysiology Jagiellonian University Medical College 18 Czysta St

31-121 Cracow, Poland Phone/fax: +48 126333947 E-mail: lukaszd@mp.pl

\begin{abstract}
Introduction: Two main pathophysiological concepts of overactive bladder (OAB) are postulated: the neurogenic and myogenic theories. Autonomic nervous system (ANS) dysfunction is also involved in OAB pathophysiology. The purpose of our study was to estimate ANS activity by heart rate variability (HRV) assessment in two $O A B$ experimental models evoked by cyclophosphamide administration: acute $(A O A B)$ and chronic $(C O A B)$ overactive ones.

Material and methods: In the $A O A B$ model, an i.p. dose of cyclophosphamide was administered ( $200 \mathrm{mg} / \mathrm{kg}$ body weight) while the COAB model received 4 times the i.p. administration of cyclophosphamide $(75 \mathrm{mg} / \mathrm{kg}$ body weight). In each subject, after urethane anaesthesia ( $1.2 \mathrm{~g} / \mathrm{kg}$ body weight), 20-minute ECG recordings (PowerLab) were performed with subsequent HRV analysis.

Results: Most of the differences in time domain analysis parameters were insignificant, except those concerning SDNN and rMSSD $(p<0.05)$. In frequency analysis, a power decrease of all standard spectral components was revealed in both OAB groups. In AOAB, TP $(1.43 \pm 1.21$ vs. $7.92 \pm 6.22$ in control; $p<0.05)$ and VLF $(0.95 \pm 1.08$ vs. $6.97 \pm 5.99$ in control; $p<0.05)$ showed significant power decrease, whereas the COAB group was mostly characterized by LF $(0.09 \pm 0.15$ vs. $0.34 \pm 0.33$ in control; $p<0.05)$ and $\mathrm{HF}(0.25 \pm 0.29$ vs. $0.60 \pm 0.41$ in control; $p<0.05)$ decrease.

Conclusions: The ANS disturbances, found as standard spectral parameter abnormalities, were demonstrated in both $A O A B$ and $C O A B$. When this finding is analysed, together with the lack of statistically significant differences in normalized $\mathrm{nLF}$ and $\mathrm{nHF}$ powers, the VLF changes seem to play an essential role, probably reflecting the progression in bladder inflammatory changes.
\end{abstract}

Key words: overactive bladder, autonomic nervous system, heart rate variability.

\section{Introduction}

The common overactive bladder (OAB) definition was given in 2002 by the International Continence Society (ICS) as urinary urgency with or without urge incontinence, usually associated with frequency and nocturia, in the absence of proven pathological or metabolic disorders or other obvious pathology [1]. According to a 2006 OAB update to the latest terminology, the reviewed $O A B$ definition is urgency with or without urgency incontinence, usually with increased daytime frequency and nocturia [2]. This clinical entity occurs with global prevalence ranging from $8 \%$ to $14 \%$, depending on variations in studied populations including sex, age and different diagnostic criteria [3]. 
There are two main pathophysiological theories describing OAB development - myogenic and neurogenic ones. The first one considers abnormal smooth muscle myoelectrical activity as the predominant OAB pathogenetic factor. According to neurogenic background, it is postulated that OAB may result from lack of central nervous inhibition of voiding or may be secondary to afferent/efferent autonomic fibre dysfunction [4-6]. Therefore, it is important to estimate autonomic nervous system (ANS) functioning, the altered activity of which may cause a predisposition to OAB. Nowadays, it is possible to evaluate ANS activity for research purposes using multiple tests based on the cardiovascular reflexes as well as direct catecholamine measurements [7]. However, the best non-invasive method of ANS indirect assessment is the heart rate variability (HRV) study. The HRV is the temporary variation of $R-R$ intervals observed in ECG recording that is modulated by autonomic influences [7-9]. Two main types of HRV analysis may be identified: time domain [8, $10]$, based on the main parameter $\mathrm{mNN}$ [ms] and other statistically derived values; and frequency (spectral) domain $[8,11]$, estimating the power spectrum and its components (TP, VLF, LF, HF).

Nevertheless, OAB is characterized by the detrusor overreactivity (DO) phenomenon, which is the main abnormality noted in urodynamic studies (bladder motility recordings). DO can be explored using experimental OAB models. One of the best described and methodically developed is that of chemical cystitis induced by cyclophosphamide (CYP). This model, first proposed by Cox [12], is frequently used by other researchers in this field, often with minor modifications.

The purpose of the study was to estimate ANS activity by HRV analysis assessment in an animal model of OAB. We used one of the best described and explored OAB experimental models - haemorrhagic cystitis (HC) evoked by CYP.

\section{Material and methods}

\section{Ethics}

The study protocol was approved by the First Local Ethic Committee in Cracow (agreement decision 126/2010).

\section{Animals and studied groups}

The experiment was carried out using 70 female rats (Wistar). The animals were obtained from the central laboratory. On arrival at the animal house of the Pathophysiology Department the rats were allowed an acclimatization period of 1 week in groups of five per cage. The animals were housed at room temperature, with a 12-12 h day-night cycle, with standard food (Labofeed Kcynia) and ad libitum water. Cyclophosphamide (CYP), a nitrogen mustard- type chemotherapeutic agent, was injected intraperitoneally (i.p.) to produce a model of chemically induced haemorrhagic cystitis (HC). According to CYP dose and route of administration, there is a possibility of inducing an 'acute overactive bladder' (AOAB) model, or a chronic case (COAB) may be evoked [13, 14]. We conducted our study in both $A O A B$ and $C O A B$.

Thus, three groups were studied: control rats (10 animals; mean body weight: $273.3 \pm 36.0 \mathrm{~g}$ ) and the AOAB (30 rats, mean body weight: $306.8 \pm 30.4 \mathrm{~g}$ ) and COAB groups (30 rats, mean body weight: 289.3 $\pm 42.1 \mathrm{~g})$.

The number of rats in all studied groups was dependent on the entire research project; the autonomic nervous system assessment by heart rate variability was the first phase of a wider experiment. The further step was urodynamic evaluation of bladder functioning after administration of two studied agents: melatonin and agomelatine. We planned to perform urodynamic recordings in both $A O A B$ (30 rats) and COAB (30 rats) populations, divided into six subgroups (each including 10 rats), according to the OAB model and studied compound in the selected dose. Therefore, initially we decided to enrol 10 rats serving as a control group, to enable further comparison of urodynamic findings. The preliminary results of the urodynamic study mentioned above has already been published in 2011 [15].

\section{$A O A B$ - acute overactive bladder group}

The AOAB model was evoked by a single i.p. administration of cyclophosphamide (Sigma-Aldrich; $200 \mathrm{mg} / \mathrm{kg}$ body weight). According to the literature data $[13,14], 4-24 \mathrm{~h}$ after exposure an acute $\mathrm{HC}$ is developed with bladder overactivity. The indirect evidence demonstrating bladder inflammation development was haematuria observed in some studied subjects. The next day an ECG recording was performed followed by a HRV analysis. Finally, the rat was put down by pentobarbital (Morbital, Biowet, Puławy) overdose.

\section{COAB - chronic overactive bladder group}

The COAB model was caused by four times i.p. CYP administration (Sigma-Aldrich; $75 \mathrm{mg} / \mathrm{kg}$ body weight), every third day during 1 week $\left(1^{\text {st }}, 3^{\text {rd }}, 5^{\text {th }}\right.$ and $7^{\text {th }}$ day). This results in chronic chemical bladder inflammation $[13,14]$. We also observed progressive haematuria, which appeared in most COAB subjects after the last CYP dose. On the $8^{\text {th }}$ day, similarly to the AOAB group, an ECG recording was recorded and after these procedures the animal was lethally anaesthetized.

\section{Control group}

The studied animals received single (control to $A O A B$ ) or four-times (control to COAB) saline i.p. 
injection in similar volumes as those administered in $A O A B / C O A B$.

\section{HRV studies}

Under urethane anaesthesia (1200 mg/kg body weight; Sigma-Aldrich) ECG recordings were performed during 20-minute rest periods in each studied animal. This anaesthetic agent was chosen having taken into consideration the literature reports which suggested the proportional (up to the applied dose) impact on tonic activity of both sympathetic and parasympathetic ANS parts and relatively small influence on cardiac reflexes $[16,17]$. After terminating ECG registration and extrasinusal ectopics elimination, HRV analysis was performed. Standard time ( $\mathrm{mNN}$ [ms], max and min NN [ms], SDNN [ms], average HR [bpm], rMSSD) and spectral (TP, VLF, LF, HF all in [ms*ms] and normalized $\mathrm{nLF}$ and $\mathrm{nHF}$ in [n.u.]) parameters were calculated. The frequency range for respective spectral components was set as $0.18<\mathrm{VLF}$ $<0.28<\mathrm{LF}<0.78<\mathrm{HF}<3$ and commonly accepted interpretation criteria were adopted: TP reflects global ANS activity, $\mathrm{HF}$ and $\mathrm{nHF}$ reflect parasympathetic tension while $\mathrm{nLF}$ reflects a pure sympathetic one, and LF is both a sympathetic and parasympathetic measure. Results are presented as mean \pm SD.

\section{Urinary bladder assessment}

In both $A O A B$ and $C O A B$ rats, urinary bladders were red, swollen and in selected cases covered by abundant serosal petechial suffusions. Leucocyte infiltration of the bladder wall was demonstrated in both $O A B$ groups. Histological inflammatory changes were more pronounced in $\mathrm{COAB}$ rats. The control rats had normal bladders.

\section{Statistical analysis}

The statistical assessment of the results obtained was conveyed after expressing them as In values using parametric test analysis of variations (Bartlett's test) with $\alpha=0.05$. The $\mathrm{HO}$ hypothesis of equality of analysed parameter variations in the three studied populations was verified versus an alternative hypothesis $\mathrm{H} 1$ of their inequality (thus, the existence of statistically significant differences). When hypothesis $\mathrm{H} 1$ for an individual parameter for the three groups was accepted, the next statistical step was to compare the differences between two groups (control vs. $A O A B$, control vs. $C O A B$, and $A O A B$ vs. $(O A B)$ using the parametric Fisher-Snedecor test with the same $\alpha$ value.

\section{Results}

\section{HRV analysis}

Most of the obtained intergroup time HRV analysis results were insignificant, except SDNN and
rMSSD. SDNN values were the highest in the control group $(4.34 \pm 2.11)$ and were lower in both $O A B$ groups ( $3.24 \pm 2.08$ in $\mathrm{AOAB}$ and $3.66 \pm 4.04$ in $\mathrm{COAB}$ ). These differences were statistically significant $(p<0.05)$ when comparing all studied groups $(1-3)$ simultaneously and when analysing the control group with $A O A B$ and $C O A B$ separately. $r M S S D$ reached the highest value in the COAB group (2.36 \pm 5.32 ), while in $A O A B$ it was similar to the control (1.65 \pm 2.77 and $1.56 \pm 0.82$, respectively); rMSSD changes were statistically significant when analysing for all three studied populations and for comparison of the control group with $C O A B$ and the $A O A B$ group with $C O A B$.

When analysing the general trend observed in other time-domain HRV parameters, it should be emphasized that some of them (minNN and rMSSD) were increased or decreased (SDNN) in both $O A B$ populations. The basic time domain analysis parameter, $m N N$, was higher in the $A O A B$ group (171.05 $\pm 12.0 \mathrm{~ms})$ and diminished in the $C O A B$ group (163.61 $\pm 12.72 \mathrm{~ms}$ ) when compared to the control group (167.84 $\pm 14.31 \mathrm{~ms})$; non-significant.

The statistical estimation of calculated spectral HRV analysis parameters indicates intergroup differences related to all non-normalized power components. Generally, the power spectrum of TP and its components was lower in both groups with bladder overactivity $(1.43 \pm 1.21$ in $A O A B$ and $2.15 \pm 2.17$ in COAB compared to $7.92 \pm 6.22$ in control; $p<0.05$ for all groups). Compared to control, the $A O A B$ group was characterized mostly by pronounced $T P$ (see above) and VLF $(0.95 \pm 1.08)$ decrease. In COAB, both $\mathrm{HF}(0.25 \pm 0.29)$ and $\mathrm{LF}(0.09 \pm 0.15)$ reduction was clearly expressed. Contrariwise, normalized ( $\mathrm{nLF}$ and $\mathrm{nHF}$ ) spectral parameters obtained in all studied populations showed similar values without statistical significance. nLF was $33.76 \pm 19.50$ n.u. in control rats, $32.83 \pm 20.87$ n.u. in $A O A B$ and 27.54 \pm 19.61 n.u. in COAB $(p>0.05)$ while nHF reached $66.25 \pm 19.50$ n.u. in controls, $67.17 \pm 20.90$ n.u. in $A O A B$ and $72.46 \pm 19.62$ n.u. in COAB $(p>0.05)$.

The results described above are presented in Table I below.

Moreover, while analysing non-normalized spectral analysis parameter results, the proportional participation of power $A O A B$ and $C O A B$ spectra related to the control spectrum (whose power components were treated as $100 \%$ ) were calculated and presented in Figure 1 below.

\section{Discussion}

As far as we know, our research is the first study revealing autonomic nervous system disturbances in an experimental, CYP-induced OAB model. In brief, we found that the AOAB model was characterized by a deep withdrawal of VLF and TP powers (the fall in TP seems be a consequence of VLF 
Table I. Selected HRV time and frequency (spectral) domain analysis parameters

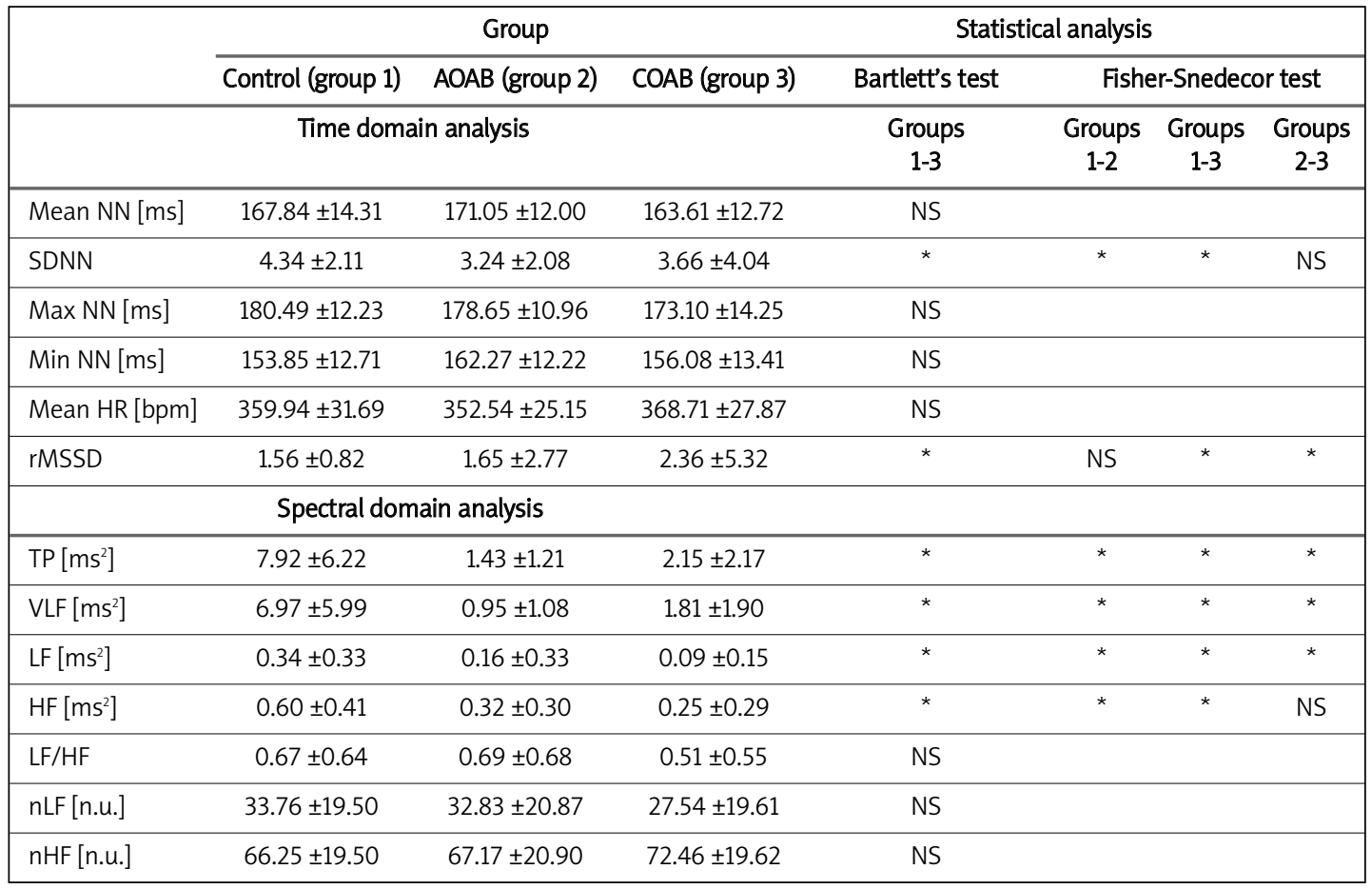

*Statistically significant, $p<0.05 ;$ NS - nonsignificant

SDNN - standard deviation of all NN intervals, rMSSD - root mean square of standard deviation of all NN intervals, TP - total power of HRV spectrum, VLF-very low frequency (component of HRV spectrum), LF-low frequency (component of HRV spectrum), HF-high frequency (component of HRV spectrum), $n L F-$ normalized LF value, $n H F-$ normalized HF value

decline because VLF mostly contributes to the TP value). Moreover, relatively high (although lower than in control) LF and HF percentages were noted, probably reflecting the intensified inflammatory bladder changes (Figure 1). In COAB, a power decrease of all spectral components was also demonstrated, but contrary to $A O A B$, the disproportions between particular components' values were not so marked in this group. Compared with $A O A B$, we observed less TP and VLF reduction, but LF and HF were more reduced (Figure 1).

The pathophysiological interpretation of the obtained results, especially those in respect to VLF, is difficult, taking into consideration that the mechanisms reflected in this spectral component are poorly understood. There is a common agreement that VLF is related to short-term sympathetic regulatory mechanisms relevant to chemoreceptors, the renin-angiotensin-aldosterone system, thermoregulatory activity and other hard-to-define phenomena $[8,9,11]$. Furthermore, there are studies suggesting that global HRV and especially VLF decrease is negatively correlated with intensification of inflammatory changes and proinflammatory cytokine levels $[18,19]$. The VLF power value reduction is considered in cardiology to be an unfavourable prognostic factor of cardiovascular complications, arising from autonomic neuropathy [20].
Our work revealed a considerable VLF decrease in AOAB subjects, which is compliant with the predictive role of this spectral component in the inflammatory changes mentioned above. The relatively high percentage of HF in the HRV spectrum may reflect the increased compensatory reaction, concerning the cholinergic anti-inflammatory pathway [21]. In COAB, the chronic character of the inflammatory process develops a smaller hypercytoki-

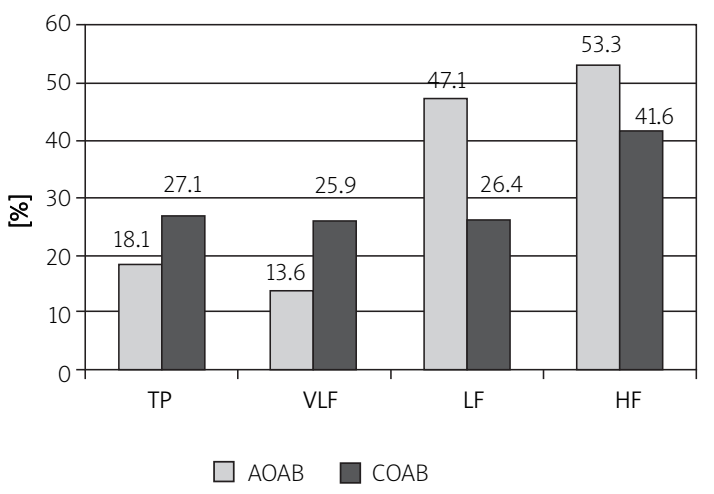

Figure 1. Percentages of HRV spectral components in OAB studied groups related to control group values treated as $100 \%$

$T P$ - total power of HRV spectrum, VLF - very low frequency (component of HRV spectrum), LF- low frequency (component of HRV spectrum), HF - high frequency (component of HRV spectrum) 
naemia level and sympathetic-vagal imbalance (expressed as VLF increase and HF decrease, compared to $A O A B)$. The interpretative difficulties become more apparent when the results demonstrating similar spectral normalized parameter values in both $\mathrm{OAB}$ and control groups are taken into consideration. In our assessment, the lack of considerable differences between normalized HRV parameters paradoxically supports the hypothesis regarding the specific character of autonomic disturbances manifesting mostly in the VLF range (which is intentionally omitted in the HRV spectrum normalization process). Further experimental and clinical investigations are needed to reveal the role of HRV spectral abnormalities in bladder inflammation and overactivity detection.

It must be emphasized that our results are consistent with the general tendency found in the few clinical studies in this research area. Choi et al. [22] reported that female $O A B$ patients were characterized by a drop in time domain parameters SDNN and rMSSD and a notable reduction in TP, VLF and $\mathrm{HF}$ powers. Our findings suggest, however, that LF power is also decreased in an experimental $O A B$ model that may be secondary to parasympathetic withdrawal and sympathetic augmentation during bladder inflammatory damage evoked by CYP. Hubeaux et al. [23] performed a study estimating the not-so-global AUN activity, as did Choi et al. [22], but the ANS activity during the filling period was evaluated in female patients with stress urinary incontinence (SUI) and idiopathic OAB (IOAB). They showed that the IOAB group was characterized by a more intense resting parasympathetic activity (demonstrated as HF power increase), while during bladder filling progressive HF reduction with LF increase was observed. Contrariwise, SUI subjects were revealed to have more stable autonomic balance in both resting and filling periods. According to Hubeaux et al. [23], in IOAB the proportions between two main ANS branches change from parasympathetic predominance in the storage period to sympathetic advantage during pre-micturition that may reflect abnormal autonomic control of bladder filling and emptying. Liao et al. [24] also found that in IOAB patients the main time domain parameter, $\mathrm{mNN}$, is lower than in control subjects. However, contrary to Choi et al. [22] they demonstrated SDNN and rMSSD increase but confirmed TP and VLF decrease in the IOAB group. Moreover, apart from HRV estimation during resting, Liao et al. [24] studied ANS variation in pre- and micturition vs. postmicturition periods. Considering the first, wider observation time they found that the control group was characterized by VLF and LF powers augmentation with small HF component representation. Recordings after bladder emptying suggested further LF predominance with no VLF presence in the HRV spectrum. In IOAB patients, considerable
HF increase with both LF and HF drops in the first observation period were revealed, and these tendencies were strengthened and deepened after micturition.

We cannot directly treat from these clinical findings due to the considerable methodological differences (we studied only resting conditions, not with reference to pre- and inter-micturition periods) and mostly because of the fact that one cannot simply compare the results obtained in clinical and experimental conditions. However, estimating global trends, our findings also support the hypothesis of autonomic disturbances in OAB course, manifesting as diminishing values of non-normalized HRV spectral components.

In conclusion, both acute (AOAB) and chronic (COAB) overactive experimental models seem to be characterized by disturbed autonomic nervous system activity manifested by diminished power spectral components in both $O A B$ groups. In the $A O A B$ group, VLF power withdrawal with relatively high LF and HF values was especially pronounced, which may suggest bladder inflammatory intensification. On the other hand, the COAB group was characterized by lower disproportions in spectral component powers; TP and VLF reached higher values while LF and $\mathrm{HF}$ reached lower ones than in $A O A B$, although all spectral components were, similarly to $A O A B$, diminished when compared to the control. Normalized frequency parameters nLF and nHF were similar in both $A O A B$ and $C O A B$; therefore it seems that the differences of VLF power (as a component that is not involved in spectrum normalization calculation) in $O A B$ groups are of great importance.

\section{References}

1. Abrams P, Cardozo L, Fall M, et al. The standardisation of terminology of lower urinary tract function: report from the Standardisation Sub-committee of the International Continence Society. Neurourol Urodyn 2002; 21: 167-78.

2. Abrams P, Artibani W, Cardozo L, Dmochowski R, van Kerrebroeck P, Sand P. Reviewing the ICS 2002 terminology report: the ongoing debate. Neurourol Urodyn 2006; 25: 293.

3. Tubaro A, Palleschi G. Overactive bladder: epidemiology and social impact. Curr Opin Obstet Gynecol 2005; 17: 507-11.

4. Hashim H, Abrams P. Overactive bladder: an update. Curr Opin Urol 2007; 17: 231-6.

5. Chu FM, Dmochowski R. Pathophysiology of overactive bladder. Am J Med 2006; 119 (3 Suppl 1): 3-8.

6. Andersson KE, Pehrson R. CNS involvement in overactive bladder: pathophysiology and opportunities for pharmacological intervention. Drugs 2003; 63: 2595-611.

7. Zygmunt A, Stanczyk J. Methods of evaluation of autonomic nervous system function. Arch Med Sci 2010; 6: 11-8.

8. Heart rate variability. Standards of measurement, physiological interpretation, and clinical use. Task Force of the European Society of Cardiology and the North American Society of Pacing and Electrophysiology. Eur Heart J 1996; 17: 354-81.

9. Rajendra Acharya U, Paul Joseph K, Kannathal N, Lim CM, Suri JS. Heart rate variability: a review. Med Biol Eng Comput 2006; 44: 1031-51. 
10. Kleiger RE, Stein PK, Bosner MS, Rottman JN. Time domain measurements of heart rate variability. Cardiol Clin 1992; 10: 487-98.

11. Ori Z, Monir G, Weiss J, Sayhouni X, Singer DH. Heart rate variability. Frequency domain analysis. Cardiol Clin 1992; 10: 499-537.

12. Cox PJ. Cyclophosphamide cystitis - identification of acrolein as the causative agent. Biochem Pharmacol 1979; 28: 2045-9.

13. Dinis P, Charrua A, Avelino A, et al Anandamide-evoked activation of vanilloid receptor 1 contributes to the development of bladder hyperreflexia and nociceptive transmission to spinal dorsal horn neurons in cystitis. J Neurosci 2004; 24: 11253-63.

14. Chopra B, Barrick SR, Meyers S, et al. Expression and function of bradykinin B1 and B2 receptors in normal and inflamed rat urinary bladder urothelium. J Physiol 2005; 562: 859-71.

15. Dobrek $Ł$, Thor PJ. The influence of melatonin and agomelatine on urodynamic parameters in experimental overactive bladder model: preliminary results. Postepy Hig Med Dosw 2011; 65: 725-33.

16. Maggi CA, Meli A. Suitability of urethane anesthesia for physiopharmacological investigations in various systems. Part 1: General considerations. Experientia 1986; 42: 109-14.

17. Maggi CA, Meli A. Suitability of urethane anesthesia for physiopharmacological investigations in various systems. Part 2: cardiovascular system. Experientia 1986; 42: 292-7.

18. Haensel A, Mills PJ, Nelesen RA, Ziegler MG, Dimsdale JE. The relationship between heart rate variability and inflammatory markers in cardiovascular diseases. Psychoneuroendocrinology 2008; 33: 1305-12.

19. Lampert R, Bremner JD, Su S, et al. Decreased heart rate variability is associated with higher levels of inflammation in middle-aged men. Am Heart J 2008; 156: 759.e1-7.

20. Hadase M, Azuma A, Zen K, et al. Very low frequency power of heart rate variability is a powerful predictor of clinical prognosis in patients with congestive heart failure. Circ J 2004; 68: 343-7.

21. Borovikova LV, Ivanova S, Zhang M, et al. Vagus nerve stimulation attenuates the systemic inflammatory response to endotoxin. Nature 2000; 405: 458-62.

22. Choi JB, Kim YB, Kim BT, Kim YS. Analysis of heart rate variability in female patients with overactive bladder. Urology 2005; 65: 1109-12.

23. Hubeaux K, Deffieux X, Ismael SS, Raibaut P, Amarenco $G$. Autonomic nervous system activity during bladder filling assessed by heart rate variability analysis in women with idiopathic overactive bladder syndrome or stress urinary incontinence. J Urol 2007; 178: 2483-7.

24. Liao WC, Jaw FS. A noninvasive evaluation of autonomic nervous system dysfunction in women with an overactive bladder. Int J Gynaecol Obstet 2010; 110: 12-7. 